\title{
A Framework for Estimating Mold Performance Using Experimental and Numerical Analysis of Injection Mold Tooling Prototypes
}

\author{
Suchana Jahan*1, Hazim El-Mounayri ${ }^{2}$, Andres Tovar ${ }^{2}$, Yung C. Shin ${ }^{1}$ \\ *Corresponding author: sjahan@purdue.edu \\ ${ }^{1}$ School of Mechanical Engineering, Purdue University, 585 Purdue Mall, West Lafayette, IN-47907 \\ ${ }^{2}$ School of Engineering and Technology, IUPUI, 723 West Michigan Street, Indianapolis, IN-46202
}

\begin{abstract}
Additive Manufacturing(AM), 3D printing, rapid prototyping, or rapid tooling refer to a range of technologies that are capable of translating virtual CAD model data into physical model. It is executed in growing number of applications nowadays. A wide range of materials are currently being used to produce consumer products and production tools. AM has brought in revolutionary changes in traditional manufacturing practices. Yet, there are certain drawbacks that hinder its advancement at mass manufacturing. High cost associated with AM is one of them. Using 3D printed tooling can provide long-time cost effectiveness and better product quality. Additively manufactured injection molds can increase the cooling performance, reduce production cycle time, and improve surface finish and part quality of the final plastic product. Yet, manufacturers are still not using the printed molds for industrial mass production. Numerical analysis can provide approximation of such improved performance, but, factual experimental results are necessary to satisfy performance criteria of molds to justify the large investment into tooling for existing industries. In this research work, a desktop injection molding machine is used to evaluate performance of 3D printed molds to develop a cost and performance analysis tool. It serves as a baseline to predict the performance of molds in real-time mass manufacturing of consumer products. The analysis describes how appropriate the estimation can be from any simulation study of molds, how much the scaling down of tool and molding system can affect the prediction of actual performance, what correction factors can be used for better approximation of performance matrices. Several "scaled down" prototypes of injection molds have been used. They have design variations as: with or without cooling system, conformal or straight cooling channels, solid or lattice matrix, and metal or tough resin as the mold material. The molds are printed in in-house printing machines and can also be printed online with limited charges. This also provides an excellent demonstration of using inexpensive material and manufacturing process, such as resin to estimate the performance of highly expensive 3D printed stainless steel molds. The work encompasses a framework to reduce overall cost of implementing AM, by lowering time and monetary expenses during the research and development, and prototyping phases.
\end{abstract}

Keywords: 3D printing, injection molding, scaled down prototype, performance comparison, conformal cooling

This is the author's manuscript of the article published in final edited form as:

Jahan, S., El-Mounayri, H., Tovar, A., \& Shin, Y. C. (2019). A Framework for Estimating Mold Performance Using Experimental and Numerical Analysis of Injection Mold Tooling Prototypes. In S. Kramer, J. L. Jordan, H. Jin, J. Carroll, \& A. M. Beese (Eds.), Mechanics of Additive and Advanced Manufacturing, Volume 8 (pp. 71-76). https://doi.org/10.1007/978-3-319-95083-9_13 


\section{INTRODUCTION}

In recent years, additive manufacturing has become the buzzword in manufacturing world. People are trying to build anything and everything with additive manufacturing, commonly known as 3D printing. With its versatile potential to build a lot of critical and complex geometry, this technology is bringing in revolutionary changes in the manufacturing industry. But cost of 3D printing becomes a major hindrance for this technology to be used in mass production.

One of the prospective fields of application of 3D printing is in the plastic injection molding industry. The current practice of this industry is to use traditional manufacturing operations, such as milling, drilling, grinding etc. to make the molding tools and use them for the final production of plastic parts in the plant. 3D printing can take over the production of tools and provide optimally designed highly effective molds that can offer long-term business benefits. But this is a difficult decision for the mold makers to move out from their traditional practice and invest into the additive manufacturing of the molding tools. Major difficulty lies in predicting and testing the mold before going into mass scale production. This situation led into trying out a low cost way to predict the mold behavior. This paper presents a solution to this situation and establishes a framework to lead the pathway towards a cost -effective analysis and prediction tool to help taking fast decisions in molding industry.

\section{METHODOLOGY}

This paper presents a basic framework towards creating an accurate performance prediction tool of plastic injection molds and overall plastic injection molding technique. With the advances in engineering and technology in present age, it is a common practice to predict the behavior of any product with numerical analysis and/or computer aided simulations before going into final production or manufacturing of the product. This is the same in the case of additive manufacturing too. But, additive manufacturing or 3D printing being a new technology, there are not yet enough industrially available simulation software packages that can predict all the steps and patterns related with this process in a comprehensive manner.

In the effort to create a comprehensive performance prediction tool, an intermediate analysis tool is needed to be developed, which can use the results available from currently existing simulation tools, match the results with experimental tests, analyze the effects of different correlated factors and finally provide a comprehensive and more accurate prediction of the process.

To predict the performance or behavior of a final product, it is possible to follow two different methods:

1) Using numerical modeling.

2) Using experimental testing on full scale or scaled down prototypes

\section{Predict using numerical modeling}

This is the method that uses numerical simulations, FEA modeling of different types to predict different types of behavior of the mold and the final product. Currently, there are different simulation tools, but they are not comprehensive for 3D printed molds. So, we can do a number of various types of simulation, do some experimental testing, and then find out a trendline on how the simulation results matches or varies from experimental output and if there is a trend on how that varies with some related factors, and if there is some correlation between the result variation and those factors. All these results can finally lead to a more accurate prediction of mold behavior.

An example of prediction using numerical modeling can be shown from published research work of the authors [1]. This includes a simple transient thermal simulation on a plastic injection mold and predicting the total cooling time. The simulation predicts that in industrial setup, a plastic bottle cap made of polypropylene will take $28.25 \mathrm{~s}$ to cool down to $50^{\circ} \mathrm{C}$. While in the experimental testing within the actual industrial circumstances, the cooling time is measured to be 30s. Though this less than 2seconds difference seem to be very insignificant, but it is very crucial for industry in mass production. It is necessary to establish a technique that reduces the $5 \%$ variation in numerical and simulation results for the industries to compete in todays business world.

\section{Predict using prototypes}

The aforementioned prediction model in the previous paragraph has an inherent difficulty within itself. To create an accurate trendline between experimental and simulation results, we need to create the full-size 3D printed molds and test them in actual industrial size injection molding machines, these are highly expensive, both the full size printed molds and also the molding machines. To minimize this difficulty, the easier prediction tool utilizes a smaller scale molding machines, small or scaled 
down molds and respective simulation tools. If anyone has a simulation model for analyzing larger molds, it's quite easy to analyze the smaller size molds too. But the difference comes in the cost savings in terms of the physical tools. This prediction technique involves identifying the experimental and numerical results of the scaled down mold, comparing them to the fullsize molds numerical and experimental results, and trying to find out whether or not there are relations between full scale and scaled down models. It is expected to find a trend on how the smaller mold behaves and how the larger mold behaves and so on. The ultimate goal is to create such a tool that can predict the behavior of full size industrial mold from a smaller size simulation and /or in house experimental results.

Using a desktop mini injection molding machine, as shown in figure 1, it is possible to experimentally test a number of scaled down 3D printed molds. In the following sections, we will try to demonstrate a framework for design of experiments that uses these techniques and finally can provide accurate predictions of mold performances.

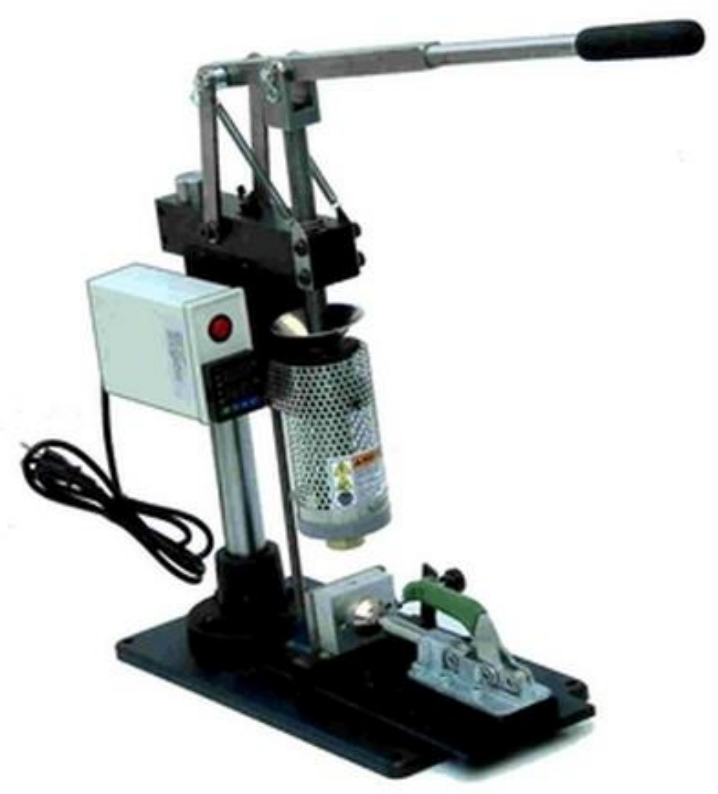

Figure 1: Mini injection molding machine

This paper is about creating the framework. Our future publications will include all the results that we can obtain using the prediction models. But we have some initial results, that can help to identify and illustrate the actual working model of the ongoing project.

\section{Design of Experiments Framework}

In the previous section, we have demonstrated just the basics of creating the comparison baseline, that can pave the way of a more comprehensive analysis framework. For this, we need a lot more results, both experimental and numerical. A number of Design of Experiments(DOE) are planned to establish a comprehensive analysis and prediction tool. The details of the design of experiments are mention below.

\section{Design of Experiment 1:}

Purpose: Identify the effect of mold material.

This DOE is designed to identify if tough resin plastic prototypes can serve as a substitute for stainless steel prototype. And if, we also need to find a trendline on the extent to which the correlation can be established. For this design of experiments, we are printing 4 sets of molds (one set of molding tool consists of two main parts; the cavity and the core).

1.1 Stainless steel mold for design 1(bottle cap).

1.2 Tough resin mold for design 1(bottle cap). 
1.3 Stainless steel mold for design 2(fishing bait).

1.4 Tough resin mold for design 2(fishing bait).

These 4 molds are of identical size and shape externally, maximum longitudinal dimension being 3 inch on one side. They can fit into a simple manual desktop molding machine and product polypropylene plastic parts. These molds have straight cooling channels inside for better cooling and part quality. While running the actual molding experiments, the cooling rates can be varied (at least 3 different flow rate of coolant) to obtain large number of data sets to ensure maximum accuracy. Hence the total number of data points would be (4 x number of different coolant flow rate).

Benefit: With sufficient number of data sets, this DOE can help to identify if 3D printed tough resin molds (low cost) can replace high cost 3D printed stainless steel ones. It will reduce the overall cost of experimental printing and predict the behavior and performance pattern of highly expensive full size industrial level stainless steel molds.

\section{Design of Experiment 2:}

Purpose: Identify the effect of mold size.

We already have a full size industrial level steel mold. It is needed to be tested against some other scaled down versions. Scale it down to 2 other sizes, such as $75 \%, 50 \%$ and $25 \%$. As a matter of fact, the first mold in DOE 1 (steel mold for design 1) is the $50 \%$ version here. The molds required in DOE 2 are:

$2.1100 \%$ size stainless steel mold for design 1

$2.275 \%$ size stainless steel mold for design 1

$2.350 \%$ size stainless steel mold for design 1

$2.425 \%$ size stainless steel mold for design 1

After we have all 4 molds, we will test them both experimentally and numerically. From previous studies, it is known to the authors that the experimental and numerical results do not match well [2-7]. There is about 5-10\% difference between them. It is required to find if there is a trend on how the variation works, and if there is a correlation between the sizing. For example, is it possible to find, say, the variation is $5 \%$ in full size molds, but $15 \%$ in $25 \%$ scaled down molds.

Benefit: The benefits are two-fold. First, by identifying how different the simulation results are from the experimental results, we can predict how the actual industrial results will be for other different molds in real life scenario. Second, if we can identify a trend between the variations at different sizes, we can just predict the performance of full size mold by from just experimental and simulations on very smaller size molds. This tremendously decreases the production cost and design and development stage cycle time.

\section{Design of Experiment 3:}

Purpose: Identify the effect of cooling channels.

This DOE is to see if a simulation tool can predict accurately how much does the cooling channel affects the cycle time and overall production cycle in injection molding. Currently existing injection molding tools are typically manufactured using traditional milling, drilling, grinding etc. operations, and most importantly cannot contain any sort of conformal cooling channels. Conformal channels have better cooling potentials for molding tools can be incorporated into mold by 3D printing process. To identify what type of cooling channel can serve the purpose best, we will design and analyze the following molds in the same overall size:

3.1 Stainless steel mold for design 1; no cooling channel.

3.2 Stainless steel mold for design 1; straight cooling channel.

3.3 Stainless steel mold for design 1; conformal cooling channel type1.

3.4 Stainless steel mold for design 1; conformal cooling channel type2. 
3.5 Tough resin mold for design 1; no cooling channel.

3.6 Tough resin mold for design 1; straight cooling channel.

3.7 Tough resin mold for design 1; conformal cooling channel type1.

3.8 Tough resin mold for design 1; conformal cooling channel type2.

These molds can be tested both experimentally and numerically. Moreover, in numerical analysis we can run a number of tests with varying designs of conformal channels size, shape and cross section and hence identify the optimum design.

Benefits: This DOE will provide in-depth information on the optimum cooling technique for injection molds. With sufficient resource and technique time, it is possible to analyze very different design and options of cooling and provide design specific optimal design rules for the mold designers.

\section{Design of Experiment 4:}

Purpose: Identify the effect of coolant fluid.

Experimentally we can use only a few coolants due to time and resource limitations. But while applying computer aided engineering, it is possible to test a number of coolants. The basic idea is to run experiments and simulations for limited number of molds (4) and find a trend on how the results relate and then based on other simulations with other coolants, predict the behavior and performance of those coolants to be used in practical industrial setup.

Following 3 molds will be used for experimental testing, with compressed air and water, hence 6tests in this DOE.

4.1 Stainless steel mold for design 1; straight cooling channel.

4.2 Stainless steel mold for design 1; conformal cooling channel type1.

4.3 Stainless steel mold for design 1; conformal cooling channel type2.

Benefits: This DOE provides significant information on the properties, application, advantages and disadvantages, feasibility and behavioral predictability of different number of coolants in injection molding, which cannot be tested in practical scenario. Once we can establish that one fluid is optimum and feasible in certain condition, it becomes much easier from the business perspective to take any investment decision, such as changing cooling system in the industry.

\section{Design of Experiment 5:}

Purpose: Identify the effect of plastic material (the one for the final product).

This DOE is based on traditional simulation technique. To predict the behavior of a final product, it is common practice to create a simulation of the same and test it. For example, if a polypropylene plastic ball needs to be manufactured using industrial plastic injection molding machine, we should first simulate the same conditions in a computer aided simulation scenario and see how it works and take significant design decisions based on that. So, in this DOE a number of popular a highly consumer plastic materials are tested in simulation scenario and predict their behavior in industrial level. Moreover, with the help of inhouse desktop molding machine, we can use an intermediate prediction tool to estimate the products performance more accurately. Hence this DOE calls for both experimental and numerical techniques. The following molds are used for experimental study:

5.1 Stainless steel mold for design 1(bottle cap).

5.2 Tough resin mold for design 1(bottle cap).

5.3 Stainless steel mold for design 2(fishing bait).

5.4 Tough resin mold for design 2(fishing bait).

Benefits: They is similar to the ones achieved in DOE. In place of coolants, it predicts the behavior of plastic product. And can demonstrate the performance of many different plastic types without ding any inhouse experiments and hence proves to be highly cost effective and less time consuming. 


\section{Conclusion}

Although this paper does not contain any concrete technical results, it provides a working principle and framework for design of experiments which is expected to be very helpful for the plastic injection molding industry. The authors are currently working on the project and will discuss the results and findings of their work in future publications. With the limitation of available simulation tools and no source of data of actual industrial level usage of 3D printed injection molds being available yet, these DOEs will be able to provide significant information and suitable guidelines for the mold designers and molding industry.

\section{References:}

1. Jahan, Suchana Akter. Optimization of conformal cooling channels in 3D printed plastic injection molds. Diss. Purdue University, 2016.

2. Wu, Tong, et al. "A framework for optimizing the design of injection molds with conformal cooling for additive manufacturing." Procedia Manufacturing 1 (2015): 404-415.

3. Jahan, Suchana A., and Hazim El-Mounayri. "Optimal Conformal Cooling Channels in 3D Printed Dies for Plastic Injection Molding." Procedia Manufacturing 5 (2016): 888-900.

4. Jahan, Suchana A., et al. "Implementation of conformal cooling \& topology optimization in 3D printed stainless steel porous structure injection molds." Procedia Manufacturing 5 (2016): 901-915.

5. Jahan, Suchana A., et al. "Thermo-mechanical design optimization of conformal cooling channels using design of experiments approach." Procedia Manufacturing 10 (2017): 898-911.

6. Wu, Tong, et al. "Design optimization of plastic injection tooling for additive manufacturing." Procedia Manufacturing 10 (2017): 923-934.

7. Jahan, Suchana A., et al. "Effect of Porosity on Thermal Performance of Plastic Injection Molds Based on Experimental and Numerically Derived Material Properties." Mechanics of Additive and Advanced Manufacturing, Volume 9. Springer, Cham, 2018. 55-63. 